\title{
The effects of social pressure on false memories
}

\author{
MatThew B. ReYSEN \\ University of Mississippi, University, Mississippi
}

\begin{abstract}
The present experiments were designed to examine the effects of social pressure on false memories. Participants studied lists created to elicit false memories and then worked in conjunction with virtual confederates on a recognition memory task. In Experiment 1, participants worked with one or two confederates to complete multiple study-test trials. On the group tests, participants were implicitly pressured to recognize words that did not appear on the studied lists. Experiment 2 was implemented similarly, but utilized a presumably more difficult recognition test involving one long study phase followed by one long test phase. After the purported group tests in both experiments, participants completed surprise individual recognition tests. In both experiments, social pressure influenced participants' responses on group recognition tests and subsequent individual recognition tests. Furthermore, the results indicated that social pressure affected both veridical memories and false memories.
\end{abstract}

It has been known for many years that social influences are capable of influencing memory performance (see, e.g., Allen \& Bragg, 1968; Deutsch \& Gerard, 1955; see Weldon, 2001, for a review). Recently, a number of studies have demonstrated a range of situations in which such effects have been observed (e.g., Bless, Strack, \& Walther, 2001; Reysen, 2003; Schneider \& Watkins, 1996). The majority of these studies have employed either a collaborative inhibition paradigm (e.g., Basden, Basden, Bryner, \& Thomas, 1997; Weldon \& Bellinger, 1997) or a social contagion paradigm (e.g., Roediger, Meade, \& Bergman, 2001). In studies examining collaborative inhibition, group recall is typically worse than the pooled recall of an equal number of participants working alone (Weldon \& Bellinger, 1997). In studies employing a social contagion paradigm (Basden, Reysen, \& Basden, 2002; Betz, Skowronski, \& Ostrom, 1996; Bless et al., 2001; Hoffman, Granhag, See, \& Loftus, 2001; Meade \& Roediger, 2002; Mudd \& Govern, 2004; Roediger et al., 2001), participants complete memory tests in the presence of a confederate who provides misleading information. Later, participants are tested individually and often incorporate some of the misleading information provided by the confederate into their own version of events.

Studies employing a social contagion paradigm provide valuable insights into the manner in which participants incorporate into their own memory reports memories purportedly produced by others. However, despite the fact that many social contagion studies are framed, at least in part, in the context of traditional conformity experiments (e.g., Asch, 1952; Sherif, 1937), researchers have largely ignored the effects of social pressure on memory performance. More specifically, the majority of studies examining social influences on memory performance have failed to place any explicit or implicit pressure on the participant to incorporate the confederate's version of events into the participant's own memory report. Instead, such studies typically provide the participant with additional, sometimes misleading, information regarding an original event, and subsequently examine the impact of that misleading information on participants' later memory performance.

The few studies that have examined the effects of social pressure on memory performance have demonstrated that such pressure can affect performance. For example, Schneider and Watkins (1996) examined the effects of social pressure on recognition judgments. Participants studied a list of words and then completed a recognition test with the assistance of a confederate. For each word on the recognition test, both the participant and the confederate determined whether a target item was on the original list or not. Sometimes the participant responded first; at other times the confederate did. When the participant responded second, he or she presumably faced some degree of implicit pressure to agree with the confederate's responses, which were manipulated through subtle cues provided by the experimenter; sometimes, however, the confederate intentionally responded incorrectly. When the participants responded second, their responses often conformed to the confederate's initial response. This basic result has since been replicated using different stimuli (Wright, Self, \& Justice, 2000).

Whereas these studies provide evidence that participants' recognition performance can be affected by the presence of a confederate, they do not provide clues to whether social pressure can affect participants' subsequent individual memory reports. To address this issue, Reysen (2005) essentially replicated Schneider and Watkins's (1996) study, with the addition of a final individual 
recognition test following the group test. Using virtual confederates instead of real confederates (see Crutchfield, 1955), participants often conformed in their responses to both correct and incorrect responses on an initial group test. In addition, the participants' performance on the final individual recognition test was affected by the initial group interaction. When participants had conformed in their responses to incorrect confederate responses on the group test, they were more likely to make subsequent recognition errors when responding in isolation on the final recognition test. Thus, initially working with an incorrect confederate affected the participants' subsequent individual recognition performance.

The purpose of the present experiments was to determine whether social pressure is capable of influencing participants' false memory rates; in other words, can people be pressured into forming or avoiding false memories? In the present experiments, participants studied word lists designed to elicit false memories (see Roediger \& McDermott, 1995) and subsequently worked in conjunction with virtual confederates on recognition memory tests. Experiment 1 was designed to incorporate implicit pressure to choose an obviously incorrect response, a critical aspect of Asch's (1952) conformity studies. This was accomplished by having participants study relatively short word lists followed by brief recognition tests. On some recognition tests, participants worked in conjunction with a virtual confederate who occasionally provided incorrect responses with which the participant was forced to either agree or disagree. The effect of this manipulation on subsequent individual recognition performance was examined. In Experiment 2, participants completed a similar task, but the recognition test was presumably more difficult, because, rather than complete multiple study-test trials, participants completed one long study phase followed by a test phase. In both experiments, the level of implicit pressure to agree with confederates was manipulated by having participants respond in one of three conditions: alone, in conjunction with one virtual confederate, or in conjunction with two virtual confederates. It was presumed that there would be more implicit social pressure to agree with two confederates than with one (Asch, 1952).

\section{EXPERIMENT 1}

The purpose of Experiment 1 was to determine whether social pressure could affect participants' false memory rates under conditions akin to those faced by participants in Asch's (1952) conformity experiments. Participants studied 10 lists composed of 12 strong associates to critical lures drawn from Stadler, Roediger, and McDermott's (1999) norms. Using a between-participants manipulation, each participant completed a recognition test following every study trial in one of three conditions: alone, with one virtual confederate, or with two virtual confederates (see Crutchfield, 1955; Reysen, 2005). During the group recognition tests, the virtual confederates responded first and the participant responded last. The virtual confederates' responses were predetermined, and the virtual confederates falsely recognized all of the critical lures. After completing 10 study-test trials, each participant completed a surprise final individual recognition test. Participants' false memory rates, as well their responses to studied words, were examined both on the initial tests that purportedly involved other participants and on the final individual test.

\section{Method}

Participants, Design, and Materials. The participants were 135 undergraduate psychology students who participated in fulfillment of partial course requirements. The experiment consisted of a $3 \times$ $4 \times 2$ mixed design. The levels of social pressure (no confederate, one confederate, or two confederates) were manipulated between participants. The status of the recognition test items (studied word, critical lure, related but nonpresented lure, and unrelated and nonpresented lure) was manipulated within participants, as was the accuracy of the confederate responses (correct or incorrect). The primary dependent variable was the proportion of test items participants correctly identified as either "old" or "new" on both the group recognition tests and the individual recognition test.

The experiment consisted of 10 study-test trials followed by a surprise final individual recognition test. On each of the 10 study-test trials, participants studied a 12-item word list drawn from Stadler et al.'s (1999) norms. Following this, participants in the social influence conditions completed a 16-item group recognition test. Participants working alone completed an identical test in isolation. After completing the 10th trial of this type, all participants completed a 160 -item final individual recognition test. The final individual recognition test consisted of each of the original 16-item recognition tests in their original order.

Procedure. Participants arrived at the laboratory alone, in pairs, or in groups of three. On arriving, they signed an informed consent form and then listened to the instructions, which informed them that they were going to work to remember several lists of words. Participants in the social influence conditions were informed that they would be working in conjunction with either one or two other participants to complete this task. All participants were told that, after studying each word list, they would solve several simple addition and subtraction problems. Next, they were told that they would be asked to complete a recognition test during which words would appear on the screen one at a time. Participants were further informed that their job was to determine whether each word that appeared on the screen was "old"- that is, on the original list—or "new"- - not on the original list. The participants were instructed that when it was their turn to respond, an arrow would appear on the screen. Participants in the social influence conditions were told that all participants would respond to each item, and that throughout the experiment they would take turns in a randomly determined order; in fact, each participant in the social influence conditions responded last. Participants in the social influence conditions were also instructed to do their best to provide the correct response on every trial, regardless of their group members' responses.

After listening to the instructions, each participant was led to a separate testing room and seated in front of a personal computer. Participants in the social influence conditions were erroneously told that the computers were linked to a network. They were also told that responses typed on an individual computer would be visible on the other computers, and vice versa. In an attempt to increase the effectiveness of the deception, cords were run from the back of each computer through the wall into adjacent rooms. After any final questions were answered, the study-test phase of the experiment began with a list of 12 words presented one at a time on a computer monitor at a rate of $1.5 \mathrm{sec}$ per word. Following the presentation of the last word in each list, for $10 \mathrm{sec}$ participants completed simple addition and subtraction problems, then completed a 16-item recognition test consisting of 9 randomly chosen studied items, 3 nonpresented but semantically related lures, 3 nonpresented semantically unrelated lures, and 1 critical lure. The nonpresented but related lures were 
drawn from the unused portion of each list. The unrelated items were drawn from unused lists.

Each recognition test began with the presentation of a word in the middle of the computer monitor. Following this, in the social influence conditions, each participant saw the responses of other group members before having an opportunity to respond. The virtual confederates' responses ( $\mathrm{O}$ or $\mathrm{N}$ ) appeared on the screen following a random time period ranging from 2 to $4 \mathrm{sec}$. When it was the participant's turn to respond, an instruction was displayed to type either an $\mathrm{O}$ for old or an $\mathrm{N}$ for new. After the participant typed a response and pressed the Enter key, the previous responses were erased and the next word appeared; then, after the participant typed a response for the 16th item, the next study-test trial began.

In the social influence conditions, the virtual confederates' responses were manipulated according to a preset schedule. In cases in which participants worked with one virtual confederate, the confederate randomly provided an incorrect response for one studied item ("new" instead of "old"), one nonpresented but related lure ("old" instead of "new"), and one unrelated lure ("old" instead of "new") in each 16-item block. The purpose of introducing these systematic errors was to increase the effectiveness of the deception. In addition, the confederate provided an incorrect response for all of the critical lures ("old" instead of "new"). In cases in which participants worked with two virtual confederates, both confederates provided an incorrect response for one studied item, one nonpresented but related lure, one unrelated lure, and the critical lure in each block. Furthermore, they disagreed on one studied item, one nonpresented but related lure, and one unrelated lure in each block. When the confederates disagreed, it was randomly determined on a trial-to-trial basis which virtual confederate (the one responding first or second) was incorrect. All of the participant responses were scored as a function of the confederates' initial responses.

After completing the 10th study-test trial, participants completed a surprise final individual recognition test. The instructions for the individual recognition test were displayed on the monitor and were similar to those provided for the initial recognition test. Participants were asked to consider each word presented on the monitor and respond "old" to original list items and "new" to words not presented on the original list. The individual recognition test consisted of the 10 original 16-item recognition tests in their original order-160 items in all. After providing 160 individual recognition responses, participants were debriefed and received credit for their participation.

\section{Results and Discussion}

The proportions of participants' correct and incorrect responses to studied words, unrelated lures, related lures, and critical lures are displayed in Table 1 . The data from the related lures and the unrelated lures are not discussed further, because their inclusion would add substantially to the length of the Results section without contributing to the overall conclusions. Experiment 1 was designed to immerse participants in a situation in which they were implicitly pressured to respond to information that was presumed to be obviously incorrect. For example, how would a participant respond when faced with two virtual confederates, both reporting that they did not remember seeing a studied word that the participant had processed only seconds before?

Group test. An ANOVA analyzing participants' recognition accuracy for studied words as a function of the number of confederates who provided incorrect responses was statistically significant $[F(2,132)=8.82, p<.0001]$. Planned comparisons revealed that the participants' recognition accuracy was worse following one incorrect confederate response than when working alone $[t(88)=4.83$, $p<.0001$ ], and worse following two incorrect confederate responses than when working alone $[t(88)=3.56, p<$ $.001]$. However, the difference in participants' performance following one incorrect confederate response versus two incorrect confederate responses was not statistically significant $[t(88)=0.18, p>.05]$. Thus, participants often mimicked the confederates' incorrect responses and reported that a recently studied word was "new."

Whereas working with confederates on trials in which they provided incorrect responses hurt participants' performance for studied words, working with confederates on trials in which they provided correct responses enhanced participants' recognition accuracy. An ANOVA conducted on participants' hit rates to studied words following correct confederate responses was statistically significant $[F(2,132)=6.36, p<.01]$. Planned comparisons revealed that participants performed better following two correct confederate responses than when working alone $[t(88)=$ $3.46, p<.001]$, and performed better following two correct confederate responses than following one correct confederate response $[t(88)=2.13, p<.05]$. However, participants' recognition performance following one correct confederate response did not differ statistically from their recognition performance when they worked alone $[t(88)=$ $1.54, p>.05]$. Thus, for studied words, working with two correct confederates tended to improve participants' recognition accuracy, relative to the other conditions.

Table 1

Proportions of Hits (H) and False Alarms (FA) as a Function of Condition and Item Type in Experiment 1

\begin{tabular}{|c|c|c|c|c|c|}
\hline & \multirow[b]{2}{*}{ No Confederates } & \multicolumn{2}{|c|}{ One Confederate } & \multicolumn{2}{|c|}{ Two Confederates } \\
\hline & & Correct & Incorrect & Correct & Incorrect \\
\hline \multicolumn{6}{|c|}{ Initial Test } \\
\hline H-studied & .90 & .92 & .79 & .94 & .79 \\
\hline FA-unrelated & .01 & .01 & .07 & .01 & .15 \\
\hline FA-related & .07 & .05 & .22 & .05 & .27 \\
\hline FA-critical & .69 & - & .79 & - & .88 \\
\hline \multicolumn{6}{|c|}{ Final Test } \\
\hline $\mathrm{H}$-studied & .87 & .86 & .86 & .90 & .88 \\
\hline FA-unrelated & .03 & .11 & .16 & .04 & .11 \\
\hline FA-related & .21 & .35 & .31 & .24 & .35 \\
\hline FA-critical & .80 & - & .84 & - & .91 \\
\hline
\end{tabular}


An ANOVA conducted on participants' false alarm rates to critical lures as a function of the number of confederates providing incorrect responses was statistically significant $[F(2,132)=12.53, p<.0001]$. Subsequent planned comparisons revealed that participants' false alarm rates were greater in the one-confederate condition than in the no-confederate condition $[t(88)=2.27, p<$ $.05]$, and were greater in the two-confederates condition than in the no-confederate condition $[t(88)=5.16, p<$ $.0001]$. In addition, participants' false alarm rates were greater in the two-confederates condition than in the oneconfederate condition $[t(88)=2.77, p<.01]$. Thus, participants' false alarm rates to critical lures were affected by social pressure, and using two confederates had a greater impact on participants' false recognition rates than using one confederate.

Individual test. Working on trials in which confederates provided incorrect responses on the group tests did not affect participants' subsequent recognition accuracy when responding to studied words $[F(2,132)=0.46, p>$ .05]. Similarly, working on trials in which confederates provided correct responses on the group tests did not affect participants' subsequent individual recognition performance for studied words $[F(2,132)=1.79, p>.05]$. However, an ANOVA conducted on participants' false alarm rates to critical lures as a function of the number of confederates employed on the initial group tests was statistically significant $[F(2,132)=4.13, p<.05]$. Subsequent planned comparisons revealed that participants' false alarm rates to critical lures were greater when participants had initially worked with two confederates who provided incorrect responses than when participants worked alone $[t(88)=2.88, p<.05]$, and greater if participants had initially worked with two confederates who provided incorrect responses than with one confederate who provided an incorrect response $[t(88)=2.09, p<$ $.05]$. However, participants' false recognition rates were statistically equivalent when they worked initially with one confederate who provided an incorrect response or when they worked alone $[t(88)=0.76, p>.05]$. Thus, when participants worked with two confederates who provided incorrect responses on the group tests, their false alarm rates were higher on the final individual recognition test than if they had worked alone or with one confederate who provided an incorrect response.

These observations demonstrate that social pressure can affect participants' responses to both studied words and critical lures. In addition, the results seem to place some constraints on the situations in which social pressure is likely to influence recognition performance. On the group tests, participants seem more than willing to conform to information presumed to be obviously incorrect. However, the results from the final individual test suggest that, for studied words, participants do not seem to incorporate the confederates' incorrect responses into their subsequent individual memory reports. Thus, for the studied material, the participants often seem to conform when implicitly pressured, but their subsequent recognition performance is ultimately unaffected by group interaction.
A different outcome was obtained for false memories. Not only were participants likely to conform on trials to confederates who provided incorrect information - calling critical lures "old" on the group tests-but in some cases participants were more likely to incorporate these false memories into their subsequent individual memory reports. The fact that later effects on individual recognition performance were only observed when participants were implicitly pressured to conform to two confederates' incorrect version of events seems to support the idea that increasing the level of social pressure can increase the false memory rate for critical lures.

\section{EXPERIMENT 2}

One major difference between Experiment 1 and other studies that have examined the effects of social pressure on recognition performance (e.g., Reysen, 2005; Schneider \& Watkins, 1996) is that Experiment 1 used multiple study-test trials, whereas other studies have employed one long study phase followed by a test phase. This difference in methodology seems likely to have an impact on the difficulty of the recognition test, and possibly on the degree to which participants are willing to conform to confederate responses. The goal of Experiment 2 was to analyze the effects of social pressure on veridical and false recognition rates under more rigorous test conditions. In addition, the confederates in Experiment 2 sometimes correctly identified critical lures as "new." This manipulation was undertaken in an attempt to determine whether social pressure could provide an inoculation effect against the formation of false memories.

\section{Method}

Participants and Materials. The participants were 135 undergraduate psychology students who participated in fulfillment of partial course requirements. The materials were similar to those used in Experiment 1.

Design and Procedure. The design and procedure were also similar to those used in Experiment 1, but with two major differences. First, rather than using multiple study-test trials, one long 120 -item list was presented, followed by a 160 -item recognition test. The initial recognition test was completed in one of three betweenparticipants conditions: alone, with one virtual confederate, or with two virtual confederates. Following this, all participants completed a surprise final individual-recognition test. Second, in the two conditions employing confederates, five critical lures were incorrectly identified as "old" and five critical lures were correctly identified as "new." This change in methodology allowed for a determination of whether false memories could be both reduced and increased through social pressure.

\section{Results and Discussion}

Group test. The proportions of participants' correct and incorrect responses to studied words, unrelated lures, related lures, and critical lures are displayed in Table 2. Working with confederates on trials in which they provided incorrect information affected participants' accuracy when responding to studied words $[F(2,132)=$ $15.83, p<.0001]$. Planned comparisons revealed that participants performed worse following one incorrect 
Table 2

Proportions of Hits (H) and False Alarms (FA) as a Function of Condition and Item Type in Experiment 2

\begin{tabular}{|c|c|c|c|c|c|}
\hline & \multirow[b]{2}{*}{ No Confederates } & \multicolumn{2}{|c|}{ One Confederate } & \multicolumn{2}{|c|}{ Two Confederates } \\
\hline & & Correct & Incorrect & Correct & Incorrect \\
\hline \multicolumn{6}{|c|}{ Initial Test } \\
\hline H-studied & .68 & .82 & .57 & .89 & .48 \\
\hline FA-unrelated & .14 & .09 & .37 & .08 & .60 \\
\hline FA-related & .20 & .18 & .50 & .10 & .63 \\
\hline FA-critical & .72 & .43 & .84 & .30 & .89 \\
\hline \multicolumn{6}{|c|}{ Final Test } \\
\hline H-studied & .65 & .74 & .73 & .79 & .73 \\
\hline FA-unrelated & .15 & .22 & .31 & .24 & .35 \\
\hline FA-related & .27 & .44 & .50 & .33 & .50 \\
\hline FA-critical & .69 & .70 & .73 & .73 & .86 \\
\hline
\end{tabular}

confederate response than when working alone $[t(88)=$ $3.49, p<.001]$, and worse when following two incorrect confederate responses than when working alone $[t(88)=$ $6.25, p<.001]$. In addition, participants performed worse following two incorrect confederate responses than following one incorrect confederate response $[t(88)=2.12$, $p<.05]$. Thus, participants were more likely to agree with two incorrect confederates than with one incorrect confederate that a previously studied word was "new."

Similarly, working on trials in which confederates provided correct information affected participants' performance when responding to studied words $[F(2,132)=$ $58.25, p<.0001]$. Planned comparisons revealed that participants performed better following one correct confederate response than when working alone $[t(88)=7.80$, $p<.0001]$, and performed better following two correct confederate responses than when working alone $[t(88)=$ $10.88, p<.0001]$. In addition, participants performed better following two correct confederate responses than following one correct confederate response $[t(88)=2.93$, $p<.01]$. Overall, working with incorrect confederates impaired recognition accuracy for studied words, and working with correct confederates improved performance. Furthermore, regardless of the confederates' accuracy, the number of confederates employed had an impact on participants' recognition performance.

An ANOVA conducted on participants' false alarm rates to critical lures as a function of the number of confederates who provided incorrect information was statistically significant $[F(2,132)=10.85, p<.0001]$. Planned comparisons revealed that participants' false alarm rates were greater following one incorrect confederate response than when working alone $[t(88)=3.10, p<.01]$, and were greater following two incorrect confederate responses than when working alone $[t(88)=4.53, p<.0001]$. However, participants' false alarm rates were not significantly greater following two incorrect confederate responses than following one incorrect confederate response $[t(88)=$ $1.46, p>.05]$. Thus, social pressure to respond "old" to critical lures affected participants' recognition judgments for those test items.

An ANOVA conducted on participants' false alarm rates to critical lures following correct confederate responses was also statistically significant $[F(2,132)=35.04, p<$ $.0001]$. Participants' false alarm rates were lower following one correct confederate response than when working alone $[t(88)=5.96, p<.0001]$, and were lower following two correct confederate responses than when working alone $[t(88)=8.69, p<.0001]$. Furthermore, participants' false recognition rates were lower following two correct confederate responses than following one correct confederate response $[t(88)=2.33, p<.05]$. Thus, when the confederates responded correctly, increasing the implicit pressure to comply with the confederate responses decreased participants' tendency to falsely recognize critical lures.

Individual test. The final individual recognition test provided an opportunity to determine whether the conformity effects observed on the group test would carry over to a subsequent individual recognition test. Working with confederates on the group test on trials in which they responded incorrectly affected participants' subsequent recognition accuracy for studied words $[F(2,132)=6.11$, $p<.0001]$. Participants performed better after initially working with one confederate who provided an incorrect response than after working alone $[t(88)=3.07$, $p<.01]$, and better after working with two confederates who provided incorrect responses than when working alone $[t(88)=3.42, p<.001]$. However, participants' final individual recognition performance was statistically equivalent when participants initially worked with two confederates who provided incorrect responses and when participants initially worked with one confederate who provided an incorrect response $[t(88)=0.34, p>.05]$.

Working with confederates on trials in which they provided correct information on the group test also affected participants' individual recognition performance for studied words $[F(2,132)=18.66, p<.0001]$. Participants performed better after initially working with one confederate who provided a correct response than when working alone $[t(88)=4.02, p<.0001]$, and better after working with two confederates who provided correct responses than when working alone $[t(88)=6.74, p<.0001]$. However, despite an apparent trend, participants' individual recognition performance for studied words was statistically equivalent when they initially worked with two con- 
federates who provided correct responses and when they initially worked with one confederate who provided a correct response $[t(88)=1.75, p=.08]$.

An ANOVA conducted on participants' false alarm rates to critical lures on the individual test, following incorrect confederate responses on the group test, was statistically significant $[F(2,132)=8.03, p<.001]$. Subsequent planned comparisons revealed that participants' false alarm rates to critical lures were greater when participants had initially worked with two confederates who provided incorrect responses than when working alone $[t(88)=$ $4.19, p<.0001]$, and greater when participants had initially worked with two confederates who provided incorrect responses than with one confederate who provided an incorrect response $[t(88)=2.82, p<.01]$. However, participants' false memory rates were statistically equivalent when they had worked initially with one confederate who provided an incorrect response and when they worked alone $[t(88)=0.97, p>.05]$. Thus, when participants worked with two confederates who provided incorrect responses on the group test, their false alarm rates were higher on the final individual recognition test than when they had worked either alone or with one confederate who provided an incorrect response. Working with correct confederates on the group tests did not have a statistically significant impact on final individual recognition performance for critical lures $[F(2,132)=0.32, p>.05]$. Thus, the inoculation effect against reporting false memories observed on the group test disappeared when participants responded individually on a subsequent recognition test.

The results obtained in Experiment 2 provide several new insights into the manner in which social pressure affects veridical memories and false memories. First, when faced with a presumably more difficult recognition test, the participants seemed more likely to conform to both correct and incorrect confederate responses. Furthermore, in Experiment 2, initial group interaction affected subsequent recognition accuracy for both studied words and critical lures. For studied words, both correct and incorrect confederate responses tended to improve participants' final recognition accuracy over that of participants who completed the initial test alone. For critical lures, participants seemed more likely to incorporate the confederates' misinformation into their own subsequent memory reports. Finally, it was observed that the level of social pressure implicitly placed on participants to respond in a given manner had an impact on participants' memory reports. This result was obtained both in a virtual group setting and on a subsequent individual recognition test.

\section{GENERAL DISCUSSION}

Recent research on the effects of social pressure on memory performance has suggested that implicit social pressure to respond in a particular manner can influence participants' public responses (Schneider \& Watkins, 1996; Wright et al., 2001). Furthermore, at least one study has demonstrated that social pressure is capable of influencing participants' subsequent individual rec- ognition performance for studied words (Reysen, 2005). The present experiments extend our understanding of the manner in which social pressure can influence recognition memory performance for veridical memories and false memories.

First, social pressure was found to influence participants' memories for studied words and critical lures. In Experiment 1, participants often agreed with confederate responses on a group test, regardless of the accuracy of those responses. However, the only evidence for the transmission of incorrect information from the confederates to participants' subsequent individual memory reports occurred when participants worked with two confederates who incorrectly called critical lures "old." In Experiment 2 , when faced with test conditions that were, presumably, more rigorous, confederate responses affected participants' performance to both studied words and critical lures on both the group test and the final individual recognition test.

Second, the results obtained in the present study suggest that, regardless of the type of recognition test item presented, increasing the level of social pressure to respond in a particular manner will often increase the likelihood that a participant will mimic confederate responses on group tests. Furthermore, in the case of critical lures, the level of implicit social pressure to respond "old" to a critical lure on a group test increases the likelihood of an "old" response on a subsequent individual recognition test. Thus, the present results suggest that participants are more likely to incorporate incorrect information into their own memory reports when implicitly pressured to do so, rather than when merely exposed to incorrect information.

Finally, prior research on the social contagion effect (Meade \& Roediger, 2002) has demonstrated that the likelihood that participants will incorporate misleading information into their own memory reports is dependent on the plausibility of the misleading information. The present results are consistent with these findings. On group tests, participants seem more than willing to agree with information that is, presumably, obviously incorrect. However, on a final individual recognition test, the transfer of incorrect information from confederates to participants is largely confined to instances in which the confederates' misinformation was plausible. Thus, the results of the present study are consistent with the following two conclusions: first, that plausible but incorrect information provided by confederates to participants can be incorporated into participants' subsequent individual memory reports; and second, that increasing the social pressure to accept plausible but incorrect information seems to increase the likelihood that participants will report that information on subsequent individual memory tests.

\section{AUTHOR NOTE}

I thank James S. Nairne, Henry L. Roediger III, and Barbara H. Basden for their helpful comments on previous versions of this article. Address correspondence concerning this article to M. B. Reysen, Department of Psychology, University of Mississippi, University, MS 38677 (e-mail: reysen@olemiss.edu). 


\section{REFERENCES}

Allen, V. L., \& BRAGG, B. W. (1968). Effect of group pressure on memory. Journal of Psychology: Interdisciplinary \& Applied, 69, 19-32.

Asch, S. E. (1952). Social psychology. Englewood Cliffs, NJ: Prentice Hall.

BASDen, B. H., BASDen, D. R., Bryner, S., \& Thomas, R. L., III (1997). A comparison of group and individual remembering: Does collaboration disrupt retrieval strategies? Journal of Experimental Psychology: Learning, Memory, \& Cognition, 23, 1176-1189.

Basden, B. H., Reysen, M. B., \& Basden, D. R. (2002). Transmitting false memories in social groups. American Journal of Psychology, 115, 211-231.

BetZ, A. L., Skowronski, J. J., \& Ostrom, T. M. (1996). Shared realities: Social influence and stimulus memory. Social Cognition, 14, 113-140.

Bless, H., Strack, F., \& Walther, E. (2001). Memory as a target of social influence? Memory distortions as a function of social influence and metacognitive knowledge. In J. P. Forgas \& K. D. Williams (Eds.), Social influence: Direct and indirect processes (pp. 167-184). New York: Psychology Press.

Crutchfield, R. S. (1955). Conformity and character. American Psychologist, 10, 191-198.

DEUTSCH, M., \& GERARD, H. B. (1955). A study of normative and informational social influences upon individual judgment. Journal of Abnormal \& Social Psychology, 51, 629-636.

Hoffman, H. G., Granhag, P. A., See, S. T. K., \& Loftus, E. F. (2001). Social influences on reality-monitoring decisions. Memory \& Cognition, 29, 394-404.

MEADE, M. L., \& RoEdiger, H. L., III (2002). Explorations in the social contagion of memory. Memory \& Cognition, 30, 995-1009.

MudD, K., \& Govern, J. M. (2004). Conformity to misinformation and time delay negatively affect eyewitness confidence and accuracy. North American Journal of Psychology, 6, 227-238.

Reysen, M. B. (2003). The effects of social pressure on group recall. Memory \& Cognition, 31, 1163-1168.

REYSEN, M. B. (2005). The effects of conformity on recognition judgements. Memory, 13, 87-94.

Roediger, H. L., III, \& MCDermott, K. B. (1995). Creating false memories: Remembering words not presented in lists. Journal of Experimental Psychology: Learning, Memory, \& Cognition, 21, 803-814.

RoEdiger, H. L., III, Meade, M. L., \& BergMan, E. T. (2001). Social contagion of memory. Psychonomic Bulletin \& Review, 8, 365-371.

SCHNEIDER, D. M., \& WATKINS, M. J. (1996). Response conformity in recognition testing. Psychonomic Bulletin \& Review, 3, 481-485.

SherIF, M. (1937). An experimental approach to the study of attitudes. Sociometry, 1, 90-98.

Stadler, M. A., Roediger, H. L., III, \& McDermott, K. B. (1999). Norms for word lists that create false memories. Memory \& Cognition, 27, 494-500.

WELDON, M. S. (2001). Remembering as a social process. In D. L. Medin (Ed.), The psychology of learning and motivation: Advances in research and theory (Vol. 40, pp. 67-120). San Diego: Academic.

Weldon, M. S., \& Bellinger, K. D. (1997). Collective memory: Collaborative and individual processes in remembering. Journal of Experimental Psychology: Learning, Memory, \& Cognition, 23, $1160-1175$.

WRight, D. B., Self, G., \& Justice, C. (2000). Memory conformity: Exploring misinformation effects when presented by another person. British Journal of Psychology, 91, 189-202.

(Manuscript received December 16, 2004; revision accepted for publication October 17, 2005.) 